\title{
Torted benign ovarian cyst in the first trimester. The gynaecologist's dilemma
}

\author{
A. Alalade $\cdot$ A. Abdelmagied
}

Received: 29 December 2009/Accepted: 26 April 2010/Published online: 13 May 2010

(C) Springer-Verlag 2010

Keywords Laparoscopy. Oophrectomy

\section{Background}

A 26-year-old primigravida woman presented at 8 weeks of gestation to the emergency gynaecology assessment unit with a several hours' history of acute onset abdominal pain unresponsive to simple analgesia. There was associated vaginal bleeding, nausea and vomiting. Examination confirmed markedly tender left iliac fossa with rebound tenderness. Inflammatory markers were normal. A pelvic ultrasound scan which was performed a week earlier, suggested left ovarian dermoid cyst of $8.5 \mathrm{~cm}$ diameter, a viable 7-week-old foetus and poorly defined right ovary.

She received opiate analgesia and antiemetic; however, her abdominal pain continued to increase in intensity. A diagnosis of torted the left ovary was established and after confirming foetal viability, the patient agreed to undergo laparoscopic left ovarian cystectomy/oophrectomy and was fully aware of the potential complications including miscarriage and laparotomy.

At laparoscopy, the left ovary was ischaemic and torted with a large dermoid cyst measuring about $10 \mathrm{~cm}$. The right ovary was normal with a possible corpus luteum. In view of the cyst size $(10 \mathrm{~cm})$, its nature (dermoid), and ovarian ischaemia during early pregnancy left oophrectomy was performed using coated vicryl ENDOLOOP $®$ (ETHICON) followed by drainage of the cyst inside $10 \mathrm{~mm}$ ENDO

A. Alalade $(\bowtie) \cdot$ A. Abdelmagied

Royal Gwent Hospital,

Cardiff Road,

Newport NP20 2UB, UK

e-mail: remialalade@gmail.com
$\mathrm{CATCH}^{\mathrm{TM}}$ bag (COVIDIEN) to aid retrieval and minimise peritoneal spillage of the cyst's contents. This was followed by peritoneal washing with $1 \mathrm{~L}$ of normal saline to reduce the risk of chemical peritonitis. The post operative period was uneventful and she was discharged approximately $24 \mathrm{~h}$ later. Histopathological assessment confirmed ovarian dermoid cyst with ovarian tissue showing ischaemic changes. Ultrasound scan at 10 weeks gestation confirmed live intrauterine pregnancy. Intermittent vaginal spotting continued until the 14th week of gestation. The rest of her pregnancy was uncomplicated leading to spontaneous labour at term and normal vaginal delivery of a healthy baby.

\section{Literature review and discussion}

Cystectomy, de-torsion or oophrectomy?

Adnexal torsion in pregnancy is rare with a reported incidence of one in 1,000 pregnancies [1]. Adnexal masses between 6 and $8 \mathrm{~cm}$ have a higher risk of torsion compared with other sizes with about $60 \%$ of these occurring between 10 and 17 weeks gestation [2]. The risk of torsion in pregnancy is increased due to altered anatomical positioning of abdominal organs [3], and also as a result of enlarged ovaries following ovulation induction in cases of assisted conception [4].

Mathevet et al. published a case series of 42 patients with ovarian torsion in pregnancy, only five of these occurred in the first trimester with one case requiring laparoscopic oophrectomy in view of ischaemic ovary [5]. Pansky et al. in their series managed 33 cases of ovarian torsion in pregnancy. There was a re-torsion rate of $20 \%$ of the cases initially managed by de-torsion alone. 
Oophrectomy was performed in one case because of a large dermoid cyst and ovarian ischaemia.

The corpus luteum provides hormonal support to the first trimesteric pregnancy until the placenta is well developed; and its excision during the first trimester can lead to miscarriage. Visual or ultrasonic assessment of the ovaries cannot always be used to reliably establish the presence or absence of a corpus luteum. Laparotomy was described as the most consistent method for such assessment and does offer the benefit of manual palpation and more tactile feedback [6]. Therefore, gynaecologists should avoid surgery on ovarian cysts in the first trimester whenever possible; and must inform their patients of the risk of miscarriage should surgery become unavoidable.

Traditionally, gynaecologists preferred cystectomy to preserve ovarian function with recourse to oophrectomy in cases of ovarian ischaemia. In recent years, there have been some published reports to support a conservative "detorsion" approach even with apparent ovarian ischaemia to be followed by elective cystectomy after recovery of the ovarian tissue "interval cystectomy" should the cyst persist; however, most of these were on non-pregnant cases. Oelsner et al. described a series of 102 benign torted adnexal masses (cyst types not identified) that were managed either by detorsion alone, aspiration+detorsion or primary cystectomy [7]. In this series, there was ultrasonic evidence of preserved ovarian function in $92.3 \%$ of the cases managed conservatively (detorsion alone or with aspiration), while ovarian function was lost in over $70 \%$ of the small group that underwent primary ovarian cystectomy. Primary ovarian cystectomy is therefore not recommended in cases of ischaemic ovaries in view of poorly developed lines of cleavage between the different tissues, which leads to inadvertent loss of ovarian tissue. Furthermore, detorsion alone carried $5.4 \%$ risk of retorsion prior to the planned interval cystectomy, requiring further emergency intervention [7].

Outside pregnancy conservative management should therefore be considered in cases of simple torted ovarian cysts aiming at preserving ovarian function; however, patients must be informed of the possibility of interval cystectomy should the cyst persist and the risk of re-torsion necessitating emergency surgery. As dermoid cysts do not resolve spontaneously and are likely to continue growing in size with more potential for re-torsion; cystectomy or oophrectomy is probably superior to simple de-torsion in these cases.

During pregnancy, the risk of re-torsion following primary de-torsion is significant at $20 \%$ as described by Pansky et al. With the indisputable limitations of laparoscopic surgery in the third trimester, laparotomy may become inevitable should re-torsion occur later in pregnancy particularly with dermoid cysts as these do not resolve spontaneously.

In our case, cystectomy on the ischaemic ovary was avoided as it invites significant risks of bleeding and/or loss of ovarian function, $>70 \%$ as demonstrated by Oelsner et al. Simple detorsion was avoided in view of the high risk of re-torsion of a persistent dermoid cyst, which will necessitate further surgery in advanced pregnancy. In accordance with the patient's consent primary oophrectomy was, therefore felt to be the best practical option.

\section{Conclusion}

The management of torted ovarian cyst in pregnancy remains a dilemma. Outside pregnancy this is reasonably resolved with good evidence favouring de-torsion of the ischaemic ovary over oophrectomy or primary cystectomy to preserve ovarian function. While more studies are needed on adnexal torsion during early pregnancy, the decision whether to perform de-torsion, cystectomy or oophrectomy seems to depend on the presence or absence of ischaemia, size and nature of the ovarian cysts, surgical expertise and, more importantly, on the individual patient's views and aspirations.

Conflict of interests None.

\section{References}

1. Condous G et al (2004) Should we be examining the ovaries in pregnancy? Prevalence and natural history of adnexal pathology detected in the first trimester sonography. Ultrasound Obstet Gynecol 24:62-66

2. Yen CF et al (2009) Risk analysis of torsion and malignancy for adnexal masses during pregnancy. Fertil Steril 91(5):1895-1902

3. Hermans RHM et al (2003) Adnexal masses in pregnancy. Onkologie 26:167-172

4. Pansky M et al (2009) Maternal adnexal torsion is associated with significant risk of recurrence. J Min Inv Gyn 16(5):551-553

5. Mathevet $P$ et al (2003) Laparoscopic management of adnexal masses in pregnancy: a case series. Eur J Obstet Gynecol Reprod Biol 108(20):217-222

6. Guay P, Bedoya M (1981) A study of the equivalence between rectal palpation, laparoscopy, laparotomy and ovarian dissection for evaluation of the ovarian response of PMSG-superovulated cows. Can Vet J 22(11):353-355

7. Oelsner $G$ et al (2003) Minimal surgery for the twisted ischemic adnexal can preserve ovarian function. Hum Reprod 18(12):2599 2602 